\title{
Vermicompostagem de lodo de esgoto urbano e bagaço de cana-de-açúcar ${ }^{1}$
}

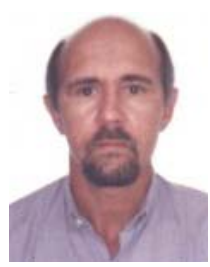

\author{
Carlos D. da Silva ${ }^{2}$, Liovando M. da Costa $^{3}$, Antonio T. de Matos ${ }^{4}$, Paulo R. Cecon ${ }^{5}$ \& Dilma D. Silva ${ }^{6}$ \\ 1 Parte da Tese de Doutorado do primeiro autor apresentada à UFV, Viçosa, MG \\ 2 UFF/MZO. R. Vital Brasil, 64, CEP 24230-340, Niterói, RJ. Fone: (21) 2682-2394. E-mail: cdomingos@ufrrj.br (Foto) \\ ${ }^{3}$ UFV/DPS. CEP 36571-000, Viçosa, MG. Fone: (31) 3899-1074. E-mail: liovando@mail.br \\ 4 UFV/DEA. Fone: (31) 3899-1420. E-mail: atmatos@mail.br \\ 5 UFV/DPI. Fone: (31) 3899-1420. E-mail: cecon@dpi.ufv.br \\ ${ }^{6}$ UFV/DPS. Bolsista de IC. E-mail: dilmadaniela@yahoo.br.com
}

Protocolo $39-2 / 4 / 2002$

\begin{abstract}
Resumo: Com o objetivo de se avaliar alterações químicas no substrato orgânico no decorrer do processo de vermicompostagem de diferentes combinações de lodo de esgoto urbano (LEU) com bagaço de cana-de-açúcar ( $\mathrm{BC}$ ), além de sua qualidade final como adubo orgânico, realizou-se um experimento em laboratório e campo. Os vermicompostos foram obtidos a partir dos tratamentos $\mathrm{T}_{1}=150 \mathrm{~kg}$ de LEU; $\mathrm{T}_{2}=121,6 \mathrm{~kg}$ de LEU $+97,30 \mathrm{~kg}$ de $\mathrm{BC} ; \mathrm{T}_{3}=59 \mathrm{~kg}$ de LEU $+97,30 \mathrm{~kg}$ de $B C ; T_{4}=35,5 \mathrm{~kg}$ de LEU $+97,30 \mathrm{~kg}$ de $B C$ e $T_{5}=23,10 \mathrm{~kg}$ de LEU $+97,30 \mathrm{~kg}$ de BC. Para a realização do processo de vermicompostagem, foram utilizadas minhocas vermelhas-da-Califórnia (Eisenia fetida). As características químicas determinadas nos vermicompostos produzidos indicaram que os mesmos podem ser utilizados como adubo orgânico, principalmente no que se refere ao conteúdo de matéria orgânica, $\mathrm{pH}$, relação $\mathrm{C} / \mathrm{N}$, concentração de nitrogênio e fósforo. As concentrações de metais pesados situaram-se abaixo dos limites de toxicidade, considerados pela legislação internacional. Aumento da proporção de LEU como substrato proporcionou aumento na concentração final de N, Ca e Mg, diminuídas de K.
\end{abstract}

Palavras-chave: lodo de esgoto urbano, biossólidos, vermicomposto, minhocas

\section{Vermicomposting of urban sewage sludge and sugarcane bagasse}

\begin{abstract}
The purpose of the present study was to evaluate the chemical alterations in the substrate with time of the vermicomposting involving different combinations of urban sewage sludge (USS) and sugarcane bagasse (SB), besides it's quality as organic fertilizer. The vermicomposts were obtained from the treatments $T_{1}=150 \mathrm{~kg}$ of USS; $T_{2}=121.6 \mathrm{~kg}$ of USS $+97.30 \mathrm{~kg}$ of SB; $T_{3}$ $=59 \mathrm{~kg}$ of USS $+97.30 \mathrm{~kg}$ of $\mathrm{SB} ; \mathrm{T}_{4}=35.5 \mathrm{~kg}$ of USS $+97.30 \mathrm{~kg}$ of SB; $\mathrm{T}_{5}=23.10 \mathrm{~kg}$ of USS $+97.30 \mathrm{~kg}$ of SB. For the vermicomposting process, Red-California earthworm (Eisenia fetida) was utilized. Chemical characteristics determined in the vermicomposts indicate that it can be used as organic fertilizer, mainly with regard to organic matter content, $\mathrm{pH}, \mathrm{C} / \mathrm{N}$ ratio, nitrogen and phosphorus levels. Heavy metal concentration of the vermicomposts was below the acceptable values recommended by international legislation. Increase of the proportion of USS as substrate increased the final concentration of $\mathrm{N}, \mathrm{Ca}$ and $\mathrm{Mg}$, and reduced that of $\mathrm{K}$.
\end{abstract}

Key words: urban sewage sludge, biosolids, vermicomposts, earthworms

\section{INTRODUÇÃO}

Os sistemas produtivos, tanto agropecuários quanto industriais e até as atividades domésticas, dão origem a resíduos orgânicos que, manejados corretamente, podem ser fonte de nutrientes para produção de alimentos, além de proporcionar melhoria das condições físicas, químicas e biológicas do solo, porém quando inadequadamente manuseados, podem constituir-se em fonte de contaminação e agressão ao meio ambi- ente. A matéria orgânica pode proporcionar a estabilização dos agregados do solo, aumentando a sua resistência à erosão e a sua capacidade de retenção de água. Matos et al. (1995) observaram aumento na capacidade de retenção de água do solo, quando da incorporação de composto orgânico ao substrato de cultivo de cenoura.

Os resíduos utilizados na vermicompostagem variam desde o lodo de esgoto de indústria de papel (Elvira et al., 1997) restos de erva-mate (Morselli et al., 1997; Quijano et al., 1997) 
lixo urbano (Venturini et al., 1999), restos de hortaliças (Dionísio et al., 1999) até finos de carvão mineral (Müller et al., 1999). Elvira et al. (1996) cultivaram Eisenia fetida e Eisenia andrei, em lodo de indústria de papel, lixo doméstico, esterco bovino e esterco de coelho. As minhocas se desenvolveram bem e apresentaram crescimento semelhante nos quatro resíduos. A capacidade reprodutiva de Eisenia fetida foi avaliada por Valente \& Morselli (1996) em diferentes substratos formados a partir da associação de várias matérias-primas, como casca de arroz, bagaço de laranja, verduras e palha seca. De acordo com Bicca et al. (1999) os vermicompostos têm carateríscticas próprias, dependendo do resíduo utilizado, de sua constituição e procedência. Ao serem associados dois ou mais resíduos orgânicos, deve-se procurar maior equilíbrio na relação $\mathrm{C} / \mathrm{N}$. Assim utiliza-se, normalmente, material rico em nitrogênio, associado a material rico em carbono orgânico. Aquino et al. (1994) estudaram a reprodução de minhocas em esterco bovino e bagaço de cana-de-açúcar e concluíram que o esterco bovino favoreceu as transformações do bagaço, provavelmente por ser fonte de nitrogênio para auxiliar a sua decomposição. Os resíduos podem ser transformados no processo de vermicompostagem em fonte de nutrientes, tanto para a produção agrícola quanto para produzir minhocas que podem ser utilizadas na alimentação animal (Vieira, 1997). Como conseqüência das transformações químicas e físicas desses resíduos, são formadas substâncias húmicas, as quais representam a maior parte da matéria orgânica do solo (Benites, 1998).

Objetivou-se, com este trabalho, avaliar alterações químicas no substrato no decorrer do processo de vermicompostagem de diferentes combinações de lodo de esgoto urbano (LEU) com o bagaço de cana-de-açúcar (BC) além de sua qualidade final como adubo orgânico.

\section{MATERIAL E MÉTODOS}

O presente trabalho foi desenvolvido na UFV, no período de $1^{\circ}$ de fevereiro a 30 de setembro de 1998, utilizando-se, como substrato, o lodo de esgoto urbano (LEU), oriundo da Companhia Estadual de Águas e Esgotos do Rio de Janeiro (CEDAE$\mathrm{RJ}$ ), associado ao bagaço de cana-de-açúcar (BC), oriundo da Usina Jatiboca, Urucânia, MG.

As matérias-primas usadas na vermicompostagem e os vermicompostos obtidos no experimento foram analisados para sua caracterização química. A determinação do pH em água foi realizada conforme metodologia descrita pela EMBRAPA (1997). A determinação do nitrogênio total foi realizada segundo o método Kjeldahl, descrito por Silva (1990). As concentrações de fósforo, cálcio e magnésio foram determinadas de acordo com a metodologia descrita por Defelipo \& Ribeiro (1981), enquanto as concentrações de micronutrientes $(\mathrm{Cu}, \mathrm{Fe}, \mathrm{Mn}$ e $\mathrm{Zn}$ ) e de metais pesados ( $\mathrm{Cd}, \mathrm{Cr}, \mathrm{Ni}$ e $\mathrm{Pb}$ ) foram determinadas a partir de extração nítrico-perclórica seguida de leitura em espectrofotômetro de absorção atômica, segundo a metodologia adotada pelo Laboratório de Nutrição Mineral de Plantas da UFV. Os resultados obtidos na matéria-prima estão apresentados na Tabela 1.
Tabela 1. Caracterização química dos substratos utilizados na vermicompostagem

\begin{tabular}{|c|c|c|}
\hline \multirow{2}{*}{ Parâmetro } & \multicolumn{2}{|c|}{ Matéria-prima } \\
\hline & LEU & $\mathrm{BC}$ \\
\hline pH (em água) & 5,80 & - \\
\hline $\mathrm{N}\left(\operatorname{dag} \mathrm{kg}^{-1}\right)$ & 1,83 & 0,30 \\
\hline $\mathrm{P}\left(\right.$ dag $\left.\mathrm{kg}^{-1}\right)$ & 1,67 & 0,03 \\
\hline $\left.\mathrm{K}(\operatorname{dag~kg})^{-1}\right)$ & 0,47 & 0,21 \\
\hline $\mathrm{Cd}\left(\mathrm{mg} \mathrm{kg}^{-1}\right)$ & 7,28 & nd \\
\hline $\mathrm{Cr}\left(\mathrm{mg} \mathrm{kg}^{-1}\right)$ & 133,60 & 9,85 \\
\hline $\mathrm{Cu}\left(\mathrm{mg} \mathrm{kg}^{-1}\right)$ & 273,00 & 6,00 \\
\hline $\mathrm{Fe}\left(\mathrm{mg} \mathrm{kg}^{-1}\right)$ & $7.416,50$ & $2.291,30$ \\
\hline $\mathrm{Pb}\left(\mathrm{mg} \mathrm{kg}^{-1}\right)$ & 333,65 & nd \\
\hline $\mathrm{Zn}\left(\mathrm{mg} \mathrm{kg}^{-1}\right)$ & 703,83 & 215,00 \\
\hline $\mathrm{C}$ orgânico (dag $\left.\mathrm{kg}^{1}\right)$ & 27,56 & 54,25 \\
\hline Relação C/N Inicial & 15,06 & 180,83 \\
\hline Mat. Seca (dag kg $\left.{ }^{-1}\right)$ & 42,81 & 36,51 \\
\hline $\begin{array}{l}\text { LEU - Lodo de esgoto urbano; BC - ba } \\
\text { N - Método Micro-Kjeldahl (Silva, } 1990 \\
\text { P - Extrator Mehlich-1 (Defelipo \& Ribe } \\
\text { K, Cd, } \mathrm{Cr}, \mathrm{Cu}, \mathrm{Fe}, \mathrm{Pb}, \mathrm{Zn}-\mathrm{HNO}_{3} / \mathrm{HClO}_{4} \\
\text { C. orgânico - Método da calcinacãa em } \\
\text { Mat. Seca - Método da estufa a } 65^{\circ} \mathrm{C} \text { (Sil } \\
\text { dd - não detectado }\end{array}$ & de-açúcar; nd & \\
\hline
\end{tabular}

O lodo de esgoto e o bagaço de cana-de-açúcar foram misturados em diferentes proporções para serem vermicompostados, constituindo cinco tratamentos (Tabela 2) e quatro repetições, no delineamento experimental inteiramente casualizado.

Tabela 2. Massas de substratos misturadas para preparo das leiras de compostagem e concentração de metais pesados*

\begin{tabular}{|c|c|c|c|c|c|c|c|}
\hline \multirow{3}{*}{ Trat } & \multicolumn{2}{|c|}{ Substratos } & \multirow{3}{*}{$\begin{array}{c}\mathrm{C} / \mathrm{N} \\
\text { Inicial }\end{array}$} & \multicolumn{4}{|c|}{ Metais Pesados } \\
\hline & LEU & $\mathrm{BC}$ & & $\mathrm{Cd}$ & $\mathrm{Cr}$ & $\mathrm{Ni}$ & $\mathrm{Pb}$ \\
\hline & \multicolumn{2}{|c|}{$\mathrm{kg}$} & & \multicolumn{4}{|c|}{$\mathrm{mg} \mathrm{kg}^{-1}$} \\
\hline $\mathrm{T}_{1}$ & 150,00 & 0,00 & 15,06 & 7,28 & 133,65 & nd & 333,65 \\
\hline $\mathrm{T}_{2}$ & 121,60 & 97,30 & 34,28 & 4,04 & 78,62 & nd & 185,34 \\
\hline $\mathrm{T}_{3}$ & 59,00 & 97,30 & 50,34 & 2,75 & 56,58 & nd & 125,95 \\
\hline $\mathrm{T}_{4}$ & 35,50 & 97,30 & 66,45 & 1,95 & 42,94 & nd & 89,18 \\
\hline $\mathrm{T}_{5}$ & 23,10 & 97,30 & 82,77 & 1,40 & 33,61 & nd & 64,03 \\
\hline
\end{tabular}

"LEU - Lodo de esgoto urbano; BC - bagaço de cana-de-açúcar; nd - não detectado

O experimento foi instalado em um galpão aberto lateralmente, onde o material foi inicialmente disposto em camadas de 0,80 x 0,80 x 0,10 m, superpostas até atingir 1,0 m de altura. Após a fase termofílica, que durou aproximadamente $60 \mathrm{~d}$, o material foi transferido para canteiros, no mesmo galpão, com dimensões de 1,0 $\mathrm{m}$ de largura por 2,0 m de comprimento, com $0,50 \mathrm{~m}$ de altura, forrados com polietileno preto.

Após $180 \mathrm{~d}$ do início do experimento de vermicompostagem, foram coletadas amostras do material, em diferentes pontos dos canteiros do minhocário, ocasião em que foram determinados o pH em água, a capacidade de troca catiônica efetiva, CTC(t) e potencial, CTC(T), conforme metodologia descrita pela EMBRAPA (1997) além do nitrogênio total, indicado conforme metodologia já citada. Nos extratos obtidos com Mehlich 1 e solução nítrico-perclórica, foram definidas as concentrações de fósforo, cálcio e magnésio, de metais pesados $(\mathrm{Cd}, \mathrm{Cr}, \mathrm{Ni}$ e $\mathrm{Pb})$.

No material a ser vermicompostado, inocularam-se 3.000 minhocas por $0,30 \mathrm{~m}^{3}$. As minhocas utilizadas foram Oligoquetas - Lumbricidae, da espécie Eisenia fetida (minhoca-vermelhada-Califórnia). 
Os resultados foram submetidos às análises de variância, com as médias dos tratamentos comparadas com uso do teste de Tukey, ao nível 0,05 de probabilidade sendo, para isto, utilizado o programa SAEG desenvolvido pela UFV.

\section{RESULTADOS E DISCUSSÃO}

Na Tabela 3 estão apresentados os valores de pH e de CTC do material em degradação, nos diferentes estádios do processo de vermicompostagem.

Tabela 3. Valores médios do $\mathrm{pH}$ em diferentes estádios e CTC no estádio $\mathrm{C}$, durante o processo de vermicompostagem dos resíduos orgânicos, submetidos a diferentes tratamentos*

\begin{tabular}{|c|c|c|c|c|}
\hline \multirow{2}{*}{ Tratamentos } & \multicolumn{3}{|c|}{ pH nos Diferentes Estádios } & \multirow{2}{*}{$\begin{array}{c}\mathrm{CTC} \\
\left(\mathrm{cmol}_{\mathrm{c}} \mathrm{kg}^{-1}\right) \\
\mathrm{C}\end{array}$} \\
\hline & A & B & $\mathrm{C}$ & \\
\hline $\mathrm{T}_{1}$ & 4,14 & 4,73 & 6,13 & $18,27 \mathrm{a}$ \\
\hline $\mathrm{T}_{2}$ & 4,63 & 5,28 & 6,50 & $13,62 \mathrm{~b}$ \\
\hline $\mathrm{T}_{3}$ & 4,89 & 5,50 & 6,08 & $9,30 \mathrm{c}$ \\
\hline $\mathrm{T}_{4}$ & 5,06 & 5,85 & 5,83 & $8,03 \mathrm{c}$ \\
\hline $\mathrm{T}_{5}$ & 5,75 & 6,30 & 5,80 & $6,80 \mathrm{c}$ \\
\hline
\end{tabular}

*As médias seguidas pela mesma letra, na coluna, não diferem ao nível de 0,05 de probabilidade pelo teste de Tukey

Estádio A - Inoculação das minhocas (1/4/1998)

Estádio B - Encerramento da vermicompostagem (30/9/1998)

Estádio C - 90 dias após o encerramento da vermicompostagem (15/1/1998)

$\mathrm{O}$ aumento nos valores de $\mathrm{pH}$ pode estar relacionado à liberação de grupamentos aniônicos provenientes da quebra de proteínas e polissacarídeos, gerando grupamentos $\mathrm{R}-\mathrm{COOH}$, $\mathrm{R}-\mathrm{OH}$ e $\varnothing-\mathrm{OH}$ (fenólico) representando a tendência de humificação do material orgânico em decomposição.

À medida que a relação BC/LEU aumentou, a CTC diminuiu, o que pode estar associado à diminuição na proporção de lodo, mais rico que o bagaço de cana-de-açúcar em proteínas, lipídeos, amidos etc., substâncias que, quando decompostas, proporcionam a formação de maior quantidade de cargas. Os resultados obtidos estão de acordo com o que foi relatado por Harada \& Inoko (1980) e Gouveia (1995), que verificaram aumento gradual da CTC com o tempo de decomposição de materiais orgânicos.

A concentração de macronutrientes nos vermicompostos (Tabela 4) além de ocorrer em razão da natural perda de massa de material orgânico na forma de gases indica, também, capacidade das minhocas em concentrar nutrientes no vermicomposto, confirmando os resultados obtidos por Aquino (1994). Os valores de nitrogênio total variaram de acordo com a proporção lodo/bagaço, ou seja, da relação $\mathrm{C} / \mathrm{N}$ inicial das medas de vermicompostagem. $\mathrm{O}$ tratamento $\mathrm{T}_{5}$, no qual a relação $\mathrm{C} / \mathrm{N}$ inicial era 5,5 vezes maior que a estabelecida no $\mathrm{T}_{1}$, apresentou concentração de nitrogênio equivalente a $70 \%$ do valor encontrado neste tratamento, indicando a capacidade das minhocas em concentrar o nitrogênio.

Ao ingerirem o alimento, rico em matéria orgânica, as minhocas podem quebrar as estruturas de compostos orgânicos e disponibilizar o fósforo. Os valores de fósforo disponível encontrados nos vermicompostos refletem esta disponibilização e sua concentração nos substratos, estando os resultados de acordo com aqueles encontrados por Pereira et al. (1999).

Verificou-se, neste experimento, que as concentrações de potássio (Tabela 4) encontradas, foram superiores àquelas obtidas por Morselli et al. (1996) em vermicomposto de esterco de coelho e que estavam relacionadas às proporções de bagaço de cana-de-açúcar utilizadas.

As concentrações de cálcio no vermicomposto foram menores que as encontradas por Morselli \& Quijano (1998) citados por Quijano (1999), o que pode estar relacionado à capacidade das minhocas em acumularem $\mathrm{Ca}$ em suas glândulas calcíferas.

Pelos resultados apresentados na Tabela 4, verificou-se que não houve diferença significativa $(p>0,05)$ nas concentrações de magnésio, entre os tratamentos que receberam bagaço de cana-de-açúcar. As minhocas podem acumular magnésio no corpo, considerando que este elemento é um ativador enzimático, essencial ao metabolismo celular (Tolonen, 1990).

Quanto à concentração de metais pesados nos vermicompostos, foi possível observar-se, no decorrer do processo de vermicompostagem, aumento nas concentrações desses metais (Tabela 4), quando se comparam com aquelas obtidas, inicialmente, nos diferentes substratos (Tabela 1), indicando aumento da concentração dos metais com o processo de vermicompostagem e as perdas de carbono no processo.

Para a concentração de $\mathrm{Cd}$, foram observadas diferenças significativas $(\mathrm{p}<0,05)$ entre os tratamentos e os valores médios encontrados acompanharam a proporção LEU/BC nos substratos de vermicompostagem. Esses valores estão acima daqueles encontrados em lodo de esgoto, pela SANEPAR (1997).

As concentrações de $\mathrm{Cr}$ encontradas nos tratamentos $\mathrm{T}_{2} \mathrm{e}$ $\mathrm{T}_{3}$ são semelhantes às citadas por Costa (1998) em composto de lixo urbano (97,04 $\left.\mathrm{mg} \mathrm{kg}^{-1}\right)$; já Venturini et al. (1999) encontraram o valor de $43 \mathrm{mg} \mathrm{kg}^{-1}$ em vermicomposto de lixo urbano.

Tabela 4. Valores médios das concentrações de macronutrientes e de metais pesados ( $\mathrm{Cd}, \mathrm{Cr}, \mathrm{Ni}$ e $\mathrm{Pb}$ ), nos diferentes vermicompostos, no estádio B do processamento*

\begin{tabular}{|c|c|c|c|c|c|c|c|c|c|}
\hline \multirow{2}{*}{ Tratamentos } & $\mathrm{N}$ & $\mathrm{P}$ & $\mathrm{K}$ & $\mathrm{Ca}$ & $\mathrm{Mg}$ & $\mathrm{Cd}$ & $\mathrm{Cr}$ & $\mathrm{Ni}$ & $\mathrm{Pb}$ \\
\hline & \multicolumn{4}{|c|}{ dag $\mathrm{kg}^{-1}$} & \multicolumn{5}{|c|}{$\mathrm{mg} \mathrm{kg}^{-1}$} \\
\hline $\mathrm{T}_{1}$ & $2,03 \mathrm{a}$ & $1,56 \mathrm{a}$ & $1,23 \mathrm{c}$ & $0,15 \mathrm{ab}$ & $7,00 \mathrm{~b}$ & $7,72 \mathrm{a}$ & $122,03 \mathrm{a}$ & $23,16 \mathrm{a}$ & $340,92 \mathrm{a}$ \\
\hline $\mathrm{T}_{2}$ & $1,74 \mathrm{~b}$ & $1,67 \mathrm{a}$ & $2,10 \mathrm{~b}$ & $0,17 \mathrm{a}$ & $12,30 \mathrm{a}$ & $5,85 \mathrm{~b}$ & $98,00 \mathrm{~b}$ & $18,78 \mathrm{~b}$ & $265,16 \mathrm{~b}$ \\
\hline $\mathrm{T}_{3}$ & $1,64 \mathrm{bc}$ & $1,67 \mathrm{a}$ & $2,28 \mathrm{~b}$ & $0,15 \mathrm{ab}$ & $11,30 \mathrm{a}$ & $4,50 \mathrm{c}$ & $89,82 \mathrm{~b}$ & $17,04 \mathrm{bc}$ & $241,03 \mathrm{~b}$ \\
\hline $\mathrm{T}_{4}$ & $1,49 \mathrm{c}$ & $1,66 \mathrm{a}$ & $2,55 \mathrm{ab}$ & $0,13 \mathrm{bc}$ & $10,20 \mathrm{ab}$ & $3,16 \mathrm{~d}$ & $74,21 \mathrm{c}$ & $15,02 \mathrm{bc}$ & $185,47 \mathrm{c}$ \\
\hline $\mathrm{T}_{5}$ & $1,42 \mathrm{c}$ & $1,38 \mathrm{a}$ & $2,93 \mathrm{a}$ & $0,11 \mathrm{c}$ & $11,00 \mathrm{ab}$ & $2,31 \mathrm{~d}$ & $63,46 \mathrm{~d}$ & $13,67 \mathrm{c}$ & $139,26 \mathrm{~d}$ \\
\hline
\end{tabular}

As médias seguidas de pelo menos uma mesma letra, na coluna, não diferem ao nível de 0,05 de probabilidade pelo teste de Tukey. $\mathrm{P}, \mathrm{K}$ - Extraídos em Mehlich-1; Ca, Mg - Extraídos em $\mathrm{KCl}$; Cd, Cr, $\mathrm{Ni}, \mathrm{Pb}$ - Extração nítrico-perclórica 
As concentrações de $\mathrm{Pb}$ estão acima daquelas encontradas em lodo de esgoto pela SANEPAR (1997). Verificou-se que os tratamentos $\mathrm{T}_{1}, \mathrm{~T}_{4}$ e $\mathrm{T}_{5}$ diferiram $(\mathrm{p}<0,05)$ e que os tratamentos $\mathrm{T}_{2}$ e $\mathrm{T}_{3}$ foram semelhantes $(\mathrm{p}>0,05)$. Nos tratamentos com menor proporção de LEU, ou seja, com maior proporção de BC, o metal chegou a atingir o dobro da concentração inicial, fato este relacionado, provavelmente, às perdas de carbono, abordadas anteriormente. Os valores encontrados nos vermicompostos foram inferiores aos $368,11 \mathrm{mg} \mathrm{kg}^{-1}$, obtidos por Costa (1998) em composto de lixo urbano. A concentração de $\mathrm{Pb}$ no vermicomposto produzido no tratamento $\mathrm{T}_{5}$ foi inferior à encontrada por Venturini et al. (1999), ou seja, $160 \mathrm{mg} \mathrm{kg}^{-1} \mathrm{em}$ vermicomposto de lixo urbano.

As concentrações de $\mathrm{Ni}$, cujos valores extremos foram, respectivamente, de 23,16 e 13,67 $\mathrm{mg} \mathrm{kg}^{-1}$, nos tratamentos $\mathrm{T}_{1} \mathrm{e}$ $\mathrm{T}_{5}$, são inferiores àquelas descobertas por Venturini et al. (1999) em vermicomposto de lixo urbano ( $\left.32 \mathrm{mg} \mathrm{kg}^{-1}\right)$; e por Costa (1998) em composto de lixo urbano (36,50 $\left.\mathrm{mg} \mathrm{kg}^{-1}\right)$.

É importante salientar que a presença das minhocas no processo não foi suficiente para reduzir a concentração de metais pesados nos vermicompostos produzidos, mesmo se sabendo que as minhocas podem acumular metais pesados em seus tecidos (Reinecke \& Reinecke, 1996; Morgan \& Morgan, 1998).

\section{CONCLUSÕES}

1. Os vermicompostos produzidos apresentaram características químicas, tais como conteúdo de matéria orgânica, $\mathrm{pH}$, relação $\mathrm{C} / \mathrm{N}$, concentração de nitrogênio e fósforo, que indicam possibilidade de uso desses materiais como adubo orgânico, em face do valor fertilizante que apresentam.

2. O vermicomposto produzido na proporção de $1,25 \mathrm{~kg}$ de lodo de esgoto, para cada quilograma de bagaço de cana-deaçúcar, foi o que agregou maior quantidade de lodo de esgoto ao bagaço de cana-de-açúcar e apresentou melhores condições químicas para utilização agrícola viabilizando, assim, o destino para ambos os resíduos.

3. Considerando a concentração de metais pesados neste trabalho, verificou-se que qualquer um dos vermicompostos produzidos pode ser utilizado na produção agrícola.

\section{AGRADECIMENTOS}

Às Universidades Federal de Viçosa e Federal Fluminense e à CAPES, pela oportunidade de realização deste trabalho.

\section{LITERATURA CITADA}

Aquino, A.M; Almeida, D.L.; De-Polli, H. Reprodução de minhocas (Oligochaeta) em esterco bovino e bagaço de cana-de-açúcar. Pesquisa Agropecuária Brasileira, Brasília, v.29, n.2, p.161-168, 1994.

Benites, V.M. Caracterização química, orgânica e espectroscópica da matéria orgânica e suas relações com a gênese de solos da Serra do Brigadeiro, da Zona da Mata Mineira. Viçosa: UFV, 1988. 123p. Dissertação Mestrado
Bicca, A.M.O.; Siqueira, D.J.; Kalil, A.C.; Menezes, F.P. Caracterização química de diferentes vermicompostos produzidos em estação quente. In: Congresso Brasileiro, 1, e Congresso Gaúcho de Minhocultura, 3, 1999. Pelotas. Resumos... Pelotas: UFPel, 1999. p.3.

Costa, C.A. Produção de alface em cultivos sucessivos adubados com composto orgânico de lixo urbano e teor de metais pesados no solo e na planta. Viçosa: UFV, 1998. 77p. Tese Doutorado

Defelipo, B.V.; Ribeiro, A.C. Análises química do solo (Metodologia). Viçosa: UFV, 1981, 17p.

Dionísio, J.A.; Pelagia, J.G.M.; Aquino, A.M. Estudo comparativo do vermicomposto produzido e comercializado na região metropolitana de Curitiba, PR. In: Congresso Brasileiro, 1, e Congresso Gaúcho de Minhocultura, 3, 1999. Pelotas, RS. Resumos... Pelotas: UFPel, 1999. p.23.

Elvira, C.; Dominguez, J.; Briones, M.J.I. Growth and reproduction of Eisenia andrei and E. fetida (Oligochaeta, Lumbricidae) in different organic residues. Pedobiologia, Jena, v.4 n.40, p.377-384, 1996.

Elvira, C.; Sampedro, L.; Dominguez, J.; Mato, S. Sludge from paper-pulp industry with nitrogen. Soil Biology and Biochemistry, Elmsford, v.29, n.3-4, p.759-762, 1997.

EMBRAPA - Empresa Brasileira de Pesquisa Agropecuária. Serviço Nacional de Pesquisa de Solos. Manual de métodos de análises de solos. Rio de Janeiro: 1997. 212p.

Gouveia, L.C. Estudo da compostabilidade do lodo de esgoto e avaliação da distribuição química de metais pesados no decorrer do processo. Viçosa: UFV, 1995. 255p. Dissertação Mestrado

Harada, Y.; Inoko, A. The measurement of the cation exchange capacity of composts for the estimation of the degree of maturity. Soil Science and Plant Nutrition, Chyoda-Ku, v.26, n.11, p.121-134, 1980.

Matos, A.T.; Sediyama, M.A.N.; Vidgal, S.M. Características químicas, físicas e microbiológicas do solo influenciadas pela aplicação de composto orgânico ou dejeto líquido de suínos. In: Seminário Mineiro sobre Manejo e Utilização de Dejetos de Suínos, 1, 1995, Ponte Nova. Resumos... Ponte Nova: EPAMIG, 1995.

Morgan, J.E.; Morgan, J.A. The distribution and intracellular compartmentation of metals in the endogeic earthworm Aporrectodea caliginosa sampled from an unpolluted and a metal-contaminated site. Environmental Pollution, Oxford. n.99, p.167-175, 1998.

Morselli, T.B.G.A.; Cruz, L.E.C.; Pocm, D.; Pich, A.H. Efeito de diferentes resíduos no comportamento de Eisenia foetida em estação quente: eclosão. In: Congresso Gaúcho de Minhocultura, 1, 1996. Pelotas. Resumos... Pelotas: UFPel, 1996. [snp].

Morselli, T.B.G.A.; Pauletto, E.A.; Menezes, A.M.B.; Gnoatto, S.C.; Silva, D.V. Influencia de diferentes misturas de resíduos orgânicos na variação populacional de Eisenia foetida e período de produção de húmus estável. In: Congresso da Pós-Graduação em Ciências Agrárias, 1, 1997. Pelotas. Resumos... Pelotas: UFPel, 1997. v.1, p.104.

Müller, J.V.S.; Mórga, A.A.; Morselli, T.B.G.A. Viabilidade da utilização de finos de carvão como substrato para vermicompostagem. In: Congresso Brasileiro, 1; e Congresso Gaúcho de Minhocultura, 3, 1999, Pelotas. Resumos... Pelotas: UFPel, 1999. p.27. 
Pereira, B.F.; Novotny, E.H.; Mangrich, A.S. Caracterização química e por espectroscopia de RPE de húmus comerciais da Região Metropolitana de Curitiba, PR. In: Congresso Brasileiro de Ciência do Solo, 27, 1999, Brasília. Resumos... Brasília: SBCS/EMBRAPA, 1999. CD-Rom

Quijano, F.G. Uso de vermicompostos na nutrição e produção de duas cultivares de alface em ambiente protegido. Pelotas, RS: UFPel 1999. 106p. Dissertação Mestrado

Quijano, F.G.; Gnoatto, S.C.; Silva, A.C.R.; Menezes, A.M.B.; Morselli, T.B.G.A. Avaliação da atividade microbiana em diferentes vermicompostos. In: Congresso da PósGraduação em Ciências Agrárias, 1, 1997, Pelotas, RS. Resumos... Pelotas: UFPel, 1997. v.1, p.99.

Reinecke, A.J.; Reinecke, S.A. The influence of heavy metal on the growth and reproduction of the compost worm Eisenia fetida (Oligochaeta). Pedobiologia, Jena, n.40, p.439-448, 1996.

SANEPAR - Companhia de Saneamento do Paraná. Utilização agrícola do lodo de esgoto no Paraná. Curitiba, 1997. 96p.
Silva, D.J. Análises de alimentos (métodos químicos e biológicos). Viçosa: UFV, 1990. 165p.

Tolonen, M. Vitamins and minerals in health and nutrition. London: Ellis Horwood. 1990. 231p. Series in Food Science and Technology

Valente, B.; Morselli, T.B.G.A. Variação populacional de Eisenia foetida em diferentes misturas de resíduos orgânicos oriundos da propriedade rural. In: Congresso da Pós-Graduação em Ciências Agrárias, 1, 1996, Pelotas. Resumos... Pelotas: UFPel, 1996. v.1, p.105.

Venturini, S.F.; Giraca, E.M.N.; Carlosso, S.J.T.; Wiethan, M.M.; Sant, L.A. Avaliação de metais pesados em composto e vermicomposto de lixo orgânico urbano. In: Congresso Brasileiro de Ciência do Solo, 27, 1999, Brasília. Resumos... Brasília: SBCS/EMBRAPA, 1999. CD-Rom

Vieira, M.L. Produção de minhocas sobre dejeto suínos para alimentação de suínos. Viçosa: UFV. 1997. 59p. Dissertação Mestrado 